\title{
Notch signaling is active in normal mouse middle ear epithelial cells
}

\author{
XIANG LIU ${ }^{1-3 *}$, HAI-BIN SHENG ${ }^{1-3^{*}}$, RUI MA ${ }^{1-4}$, JUAN-MEI YANG ${ }^{1-3}$, WEN-WEI LUO ${ }^{1-3}$, \\ XIAO-YU YANG ${ }^{1-3}$, DONG-DONG REN ${ }^{1-3}$ and FANG-LU CHI ${ }^{1-3}$ \\ ${ }^{1}$ Department of Otology and Skull Base Surgery, Eye, Ear, Nose and Throat Hospital; \\ ${ }^{2}$ Shanghai Auditory Medical Center; ${ }^{3}$ Key Laboratory of Hearing Science, Ministry of Health; \\ ${ }^{4}$ Department of Research Center, Eye, Ear, Nose and Throat Hospital, Fudan University, Shanghai 200031, P.R. China
}

Received February 25, 2015; Accepted September 28, 2015

DOI: $10.3892 /$ etm.2016.3146

\begin{abstract}
Mucous cell metaplasia/hyperplasia in the middle ear epithelium is associated with the occurrence of otitis media with effusion during infections. However, the mechanism by which Notch signaling regulates cell fate in the middle ear epithelium is unclear. The aim of the present study was to elucidate this mechanism by investigating the localization of Notch receptors, such as Notch1 and Notch2, and Notch ligands, such as Jagged1, in the normal mouse middle ear epithelium (NMMEE) using immunofluorescence. Furthermore, the mRNA expression levels of Notch receptors and ligands were evaluated using reverse transcription polymerase chain reaction (PCR). The effects of the $\gamma$-secretase inhibitor $\mathrm{N}-[\mathrm{N}$ (3,5-difluorophenacetyl)-L-alanyl]-S-phenylglycine tert-butyl ester (DAPT) on epithelial cell proliferation were determined using 5-ethynyl-2'-deoxyuridine (EdU) staining and immunofluorescence staining of the apoptosis marker caspase- 3 and the epithelial proliferation marker pan-cytokeratine. In addition, the differentiation of the NMMEE cells was characterized by evaluating the mRNA expression levels of the mucous cell-associated genes Arg2, Muc2, Spdef, Spink4 and Tff1 using quantitative PCR. Notch1, Notch2 and Jagged1 were observed to be co-localized throughout the mouse middle ear epithelium. Furthermore, Notch1-4, Jagged1, Jagged2, D111 and Dll4 mRNAs were expressed in the NMMEE cells. The inhibition of Notch by DAPT resulted in fewer EdU-positive
\end{abstract}

Correspondence to: Dr Fang-Lu Chi or Dr Dong-Dong Ren, Department of Otology and Skull Base Surgery, Eye, Ear, Nose and Throat Hospital, Fudan University, 83 Fenyang Road, Shanghai 200031, P.R. China

E-mail: chifanglu@126.com

E-mail: dongdong_ren@163.com

${ }^{*}$ Contributed equally

Abbreviations: NMMEE, normal mouse middle ear epithelium

Key words: DAPT, notch signaling, otitis media, mucous cell metaplasia, proliferation, differentiation cells and the upregulation of the expression levels of various mucous cell-associated genes. The results indicate that DAPT suppresses the proliferation of NMMEE cells while promoting their differentiation into mucous cells. Therefore, DAPT may provide a specific therapeutic strategy for the reversal of multiple pathological processes that are associated with epithelium thickening in the middle ear.

\section{Introduction}

Otitis media (OM) is a common disease in infants and may in certain cases develop into acute otitis media (AOM) or chronic otitis media. Teele et al conducted a prospective, cohort study in 1989 and estimated that $\sim 62 \%$ of infants suffered at least one episode of AOM by the age of 1 year, and $~ 83 \%$ of infants by the age of 3 years (1). However, studies from several other countries, summarized in a report on OM research published between 2003 and 2007, have indicated a lower incidence of AOM over this age range (2). In a previous study, Ting et al (3) conducted $>10,000$ questionnaires between 2005 and 2010, which revealed that the overall prevalence of AOM among Taiwanese children $<5$ years of age was $\sim 20 \%$.

The pathogenesis of $\mathrm{OM}$ is complicated, involving numerous factors associated with the anatomy, pathology and cell biology of the middle ear, mastoid, Eustachian tube and nasopharynx (4). Increasing attention is being focused on investigating the role of infection-induced mucous cell metaplasia/hyperplasia in the middle ear epithelium, and the associated mucin hyperproduction that has been identified as a fundamental occurrence of OM with effusion (5). Cellular proliferation and differentiation are essential to this mucous cell metaplasia/hyperplasia process. However, the precise mechanisms that regulate these processes have yet to be fully elucidated, and cellular interactions are a crucial factor during OM infection

The Notch signaling pathway is a highly conserved network that regulates cell fate decisions in various tissues and organisms (6). Notch proteins are membrane-bound receptors, with the corresponding membrane bound ligands Delta-like (DIl) and Jagged. Following the binding of a ligand, the Notch intracellular domain (NICD) is cleaved by $\gamma$-secretase and translocated to the nucleus, where it transactivates target genes 
such as hairy and enhancer of split (Hes) and Hes-related repressor protein (Hey). Hes and Hey function as transcriptional repressors, suppressing the expression of downstream target genes and thereby regulating cellular proliferation and differentiation $(7,8)$.

Notch signaling is involved in various aspects of cellular regulation. Depending on the tissue and context, Notch may either restrict or promote cell fate determination. In the intestine, Notch and $\gamma$-secretase inhibitors block cellular proliferation and induce secretory cell differentiation, and the Notch signaling pathway is key to the differentiation or self-renewal of intestinal stem cells (9-11). In the human corneal epithelium, $\mathrm{N}$-[N-(3,5-difluorophenacetyl)-L-alanyl]S-phenylglycine tert-butyl ester (DAPT), a specific inhibitor of $\gamma$-secretase, has been shown to inhibit Notch signaling, thereby repressing cell proliferation and promoting the differentiation of corneal epithelial cells (12). A previous study by Blanpain et al showed that Notch functions as a regulator of epidermal differentiation, in addition to regulating the balance between proliferative basal progenitor cells and terminally differentiating suprabasal progeny cells (13). Furthermore, previous studies have indicated that Notch signaling may be involved in the proliferation and differentiation of airway epithelial cells and mammary cells $(14,15)$. However, the role of Notch signaling and the expression of signaling pathway-associated genes in the middle ear epithelium remains unclear, as does the role of Notch signaling in the regulation of middle ear epithelial cell activity. The aim of the present study was to identify the localization of Notch receptors and their ligands, including Notch1-4, Jagged1, Jagged2, Dl11, Dll3 and Dll4, in normal mouse middle ear epithelium (NMMEE) cells. Furthermore, the study aimed to elucidate whether the inhibition of Notch signaling by the $\gamma$-secretase inhibitor DAPT was able to repress cellular proliferation and promote the differentiation of NMMEE cells into mucous cells through inhibit Notch signaling. Therefore, the mRNA expression levels of the mucous cell-associated genes Spink4, Tff1, Spdef, Arg2 and Muc2 were evaluated.

\section{Materials and methods}

NMMEE cell culture and observation of cell morphology. A total of 65 male BALB/c mice (age range, 4-6 weeks; weight, $20 \mathrm{~g}$ ) were used as middle ear epithelial cell donors (Shanghai Laboratory Animal Center, CAS, Shanghai, China). The animal use protocol was approved by the Institutional Animal Care and Animal Ethics Committee of Fudan University (Shanghai, China). Mice were anesthetized with ketamine hydrochloride $(100 \mathrm{mg} / \mathrm{kg}$; Hengrui Medicine Co., Ltd., Jiangsu, China) and xylazine (10 mg/kg; Sangon Biotech Co., Ltd., Shanghai, China). The bullae were immediately removed and rinsed in phosphate-buffered saline (PBS; Thermo Fisher Scientific, Inc., Beijing, China). The middle ear mucosa was aseptically dissociated from the bony part of the bullae under a stereomicroscope (Stemi 2000-C; Carl Zeiss Jena GmbH, Jena, Germany), cut into small pieces and incubated in $0.25 \%$ trypsin (Invitrogen, Carlsbad, CA, USA) and $1.6 \mathrm{mM}$ ethylenediaminetetraacetic acid for $20 \mathrm{~min}$ at $37^{\circ} \mathrm{C}$. Digestion was terminated by adding Dulbecco's modified Eagle's medium with $10 \%$ fetal bovine serum (FBS; Invitrogen). The cellular suspension was centrifuged at $160 \mathrm{xg}$ for $5 \mathrm{~min}$. The supernatant was discarded and the pellet was resuspended in full growth medium. Cells were seeded in culture dishes $\left(\sim 10^{4}\right.$ cells per dish) coated with bovine collagen I (354231; BD Biosciences, Franklin Lakes, NJ, USA) and coverslips. These primary cells were cultured at $37^{\circ} \mathrm{C}$ in a $5 \% \mathrm{CO}_{2}$ cell culture incubator. Full growth medium, containing Ham's F-12K (Kaighn's) medium supplemented with $1.5 \mathrm{~g} / \mathrm{l}$ sodium bicarbonate, $2.0 \mathrm{mML}$-glutamine, $10 \mathrm{ng} / \mathrm{ml}$ murine epidermal growth factor (Peprotech Inc., Rocky Hill, NJ, USA), $10 \mathrm{mg} / \mathrm{ml}$ insulin-transferrin-sodium selenite supplement (BD Biosciences), $2.7 \mathrm{~g} / 1$ glucose, $500 \mathrm{ng} / \mathrm{ml}$ hydrocortisone (H0888; Sigma-Aldrich, St. Louis, MO, USA), $0.1 \mathrm{mM}$ non-essential amino acids (M7145; Sigma-Aldrich) and 4\% FBS, was changed every 2-3 days for 1 week. The cells were used for experiments during an exponential growth phase. Bright-field live cell images were acquired using a Nikon Eclipse Ti-E microscope equipped with a Nikon DS-Fi1 digital camera (Nikon Corporation, Tokyo, Japan).

Identification of the immunolocalization of Notch receptors and their ligands using immunofluorescence analysis. Complete bullae were removed and fixed in $4 \%$ paraformaldehyde for $24 \mathrm{~h}$, then the middle ear epithelial cells were dissected out by the aforementioned method. The epithelial cells were treated with $0.3 \%$ Triton X-100 (Thermo Fisher Scientific, Inc., Carlsbad, CA, USA) in PBS for $30 \mathrm{~min}$, then blocked using 10\% normal donkey serum (Jackson ImmunoResearch Laboratories, Inc., West Grove, PA, USA) in PBS for $1 \mathrm{~h}$ at room temperature. Tissues were then incubated with the following primary antibodies overnight at $4^{\circ} \mathrm{C}$ : Goat polyclonal anti-Jagged1 (1:200, sc6011; Santa Cruz Biotechnology, Inc., La Jolla, CA, USA), rabbit monoclonal anti-Notch1 (1:100; D6F11; Cell Signaling Technology, Inc., Danvers, MA, USA) and rabbit monoclonal anti-Notch2 (1:100; D76A6; Cell Signaling Technology, Inc.). After rinsing in PBS, samples were incubated with fluorescence-labeled Alexa Fluor 488 (705-295-147) or 555 (711-545-152) secondary antibodies (1:1,000; Thermo Fisher Scientific, Inc.) for $2 \mathrm{~h}$ at room temperature. For nuclear labeling, samples were additionally incubated with Hoechst stain (1:1,000; Thermo Fisher Scientific, Inc.) for $30 \mathrm{~min}$. After PBS washing, specimens were mounted on glass slides and observed using a Leica SP5 confocal laser-scanning microscope (Leica Microsystems $\mathrm{GmbH}$, Wetzlar, Germany) and images were captured at a magnification of $x 40$. Images were processed using Adobe Photoshop software (Adobe Systems, Inc., Santa Clara, CA, USA).

Evaluation of the mRNA expression levels of Notch receptors and ligands by reverse transcription polymerase chain reaction (RT-PCR) analysis. Total RNA was isolated from primary cultures of NMMEE cells by directly adding the RNA lysis buffer provided in an RNeasy Micro kit (74004; Qiagen, Hilden, Germany) after rinsing with PBS twice. RNA was purified using the kit according to the manufacturer's instructions. RNA quality and quantity were assessed using a NanoDrop 2000 Spectrophotometer (Thermo Fisher Scientific, Inc.) and $500 \mathrm{ng}$ total RNA was used for each sample. cDNA was synthesized using PrimerScript RT Master mix (RR036A; Takara Bio, Inc., Otsu, Japan) according to the manufacturer's 
Table I. Reverse transcription primer sequences.

\begin{tabular}{llc}
\hline Gene & \multicolumn{1}{c}{ Primer sequences 5' to 3' } & Product size (bp) \\
\hline Notch1 & TCAGGGTGTCTTCCAGATCCCAGCATCCACATTGTTCACC & 212 \\
Notch2 & ATGTGGACGAGTGTCTGTTGCGGAAGCATAGGCACAGTCATC & 146 \\
Notch3 & TGCCAGAGTTCAGTGGTGGCACAGGCAAATCGGCCATC & 157 \\
Notch4 & CTCTTGCCACTCAATTCCCTTTGCAGAGTTGGGTATCCCTG & 188 \\
Jagged1 & CCTCGGGTCAGTTTGAGCTGCCTTGAGGCACACTTTGAAGTA & 150 \\
Jagged2 & CAATGACACCACTCCAGATGAGGGCCAAAGAAGTCGTTGCG & 203 \\
Dll1 & CAGGACCTTCTTTCGCGTATGAAGGGGAATCGGATGGGGTT & 168 \\
Dll3 & CTGGTGTCTTCGAGCTACAAATTGCTCCGTATAGACCGGGAC & 199 \\
GAPDH & TTCCAGGCAACCTTCTCCGAACTGCCGCTATTCTTGTCCC & 102 \\
\hline
\end{tabular}

Dll, Delta-like; GAPDH, glyceraldehyde 3-phosphate dehydrogenase.

instructions. PCR was conducted using an ABI-7500 RealTime PCR System (Applied Biosystems, Foster City, CA, USA).

PCR primers for Notch 1-4 receptors and their ligands (Dl11, Dl13, Dll4, Jagged1 and Jagged2) were designed according to the gene sequences obtained from GenBank (http://www.ncbi.nlm.nih.gov/genbank/). Primer sequences and the sizes of the expected PCR products are detailed in Table I. PCR was performed using Taq polymerase (DR001B; Takara Biotechnology, Co., Ltd., Dalian, China). PCR cycling consisted of 35 cycles, each conducted as follows: Denaturation for $30 \mathrm{sec}$ at $94^{\circ} \mathrm{C}$, a $30-\mathrm{sec}$ annealing step at $57^{\circ} \mathrm{C}, 30 \mathrm{sec}$ at $72^{\circ} \mathrm{C}$ and a final extension at $72^{\circ} \mathrm{C}$ for $5 \mathrm{~min}$. Glyceraldehyde 3 -phosphate dehydrogenase (GAPDH) was used as a positive control. PCR products were subjected to electrophoresis on $2.5 \%$ agarose gel (Sangon Biotech Co., Ltd.) containing ethidium bromide and visualized under ultraviolet light.

Evaluation of cellular proliferation by labeling with 5-ethynyl-2'-deoxyuridine (EdU). The $\gamma$-secretase inhibitor DAPT (565784; EMD Chemicals, Inc., San Diego, CA, USA) was used to evaluate the function of Notch signaling in the regulation of epithelial cell differentiation and proliferation. NMMEE cells were cultured on coverslips precoated with collagen I until subconfluent. The cells were subsequently incubated in serum-free medium for $4 \mathrm{~h}$, and then incubated in medium containing 2.5 or $5 \mu \mathrm{M}$ DAPT for $24 \mathrm{~h}$. Control wells were incubated in medium containing $0.2 \%$ dimethyl sulfoxide (DMSO). EdU was used to label proliferating cells, and was detected using an EdU labeling/detection kit (C10371-1; Guangzhou Ribobio, Co., Ltd., Guangzhou, China) according to the manufacturer's protocol. EdU was added to the culture media at a concentration of $10 \mu \mathrm{M}$ simultaneously with DAPT for $24 \mathrm{~h}$.

Evaluation of apoptosis and epithelial cell proliferation using immunostaining analysis. Dual EdU and immunofluorescence staining was conducted, with EdU staining performed first, followed by immunostaining. Immunostaining was performed as described above. Primary antibodies against caspase-3 (1:100; AF835; R\&D Systems, Inc., Minneapolis, MN, USA)
Table II. Quantitative polymerase chain reaction primer sequences.

\begin{tabular}{ll}
\hline Gene & \multicolumn{1}{c}{ Primer sequences 5' to $3^{\prime}$} \\
\hline Hes 1 & CGAGCGTGTTGGGGAAATAC \\
Spink4 & GCTGAGGTCATGGCGTTGATC \\
& TTTTCATCCGGGTCAGGCAA \\
Tff1 & CAGGCCCAGGAAGAAACATG \\
& AAACAGCAACCTCTCTCCGT \\
Spdef & AACGTGCAGAAGTGGCTTT \\
& ACTTCCAGATGTCCAGGTGG \\
Arg2 & TCCCTGCCAATCATGTTCCT \\
& TAGCTTCTTCTGTCCCCGAG \\
Muc2 & ATGTCCTGACCAAGAGCGAA \\
& GACAGTCTTCAGGCAGGTCT \\
GAPDH & AGGTCGGTGTGAACGGATTTG \\
& TGTAGACCATGTAGTTGAGGTCA
\end{tabular}

GAPDH, glyceraldehyde 3-phosphate dehydrogenase.

and pan-cytokeratin (pan-CK) $(1: 100 ; 4545$; Cell Signaling Technology, Inc.) were used, overnight at $4^{\circ} \mathrm{C}$. Experiments, in triplicate, were repeated at least twice. Images were captured digitally in at least six random microscope fields from each sample, using a Leica DM 4000 B microscope equipped with a DFC 500 camera (Leica, Mannheim, Germany).

Quantitative results were obtained by calculating the ratio between the number of immunopositive cells for each antibody and the total number of cells, which determined by counting the nuclei revealed by Hoechst staining from six randomly selected microscopic fields (magnification, x400). Images were processed using Adobe Photoshop software.

Detection of the expression of mucous cell-associated genes in using quantitative RT-PCR (RT-qPCR). Epithelial cells cultured in $6-\mathrm{cm}$ dishes were treated with DAPT as described in the cell proliferation assay. Subsequently, 3-5 independent 

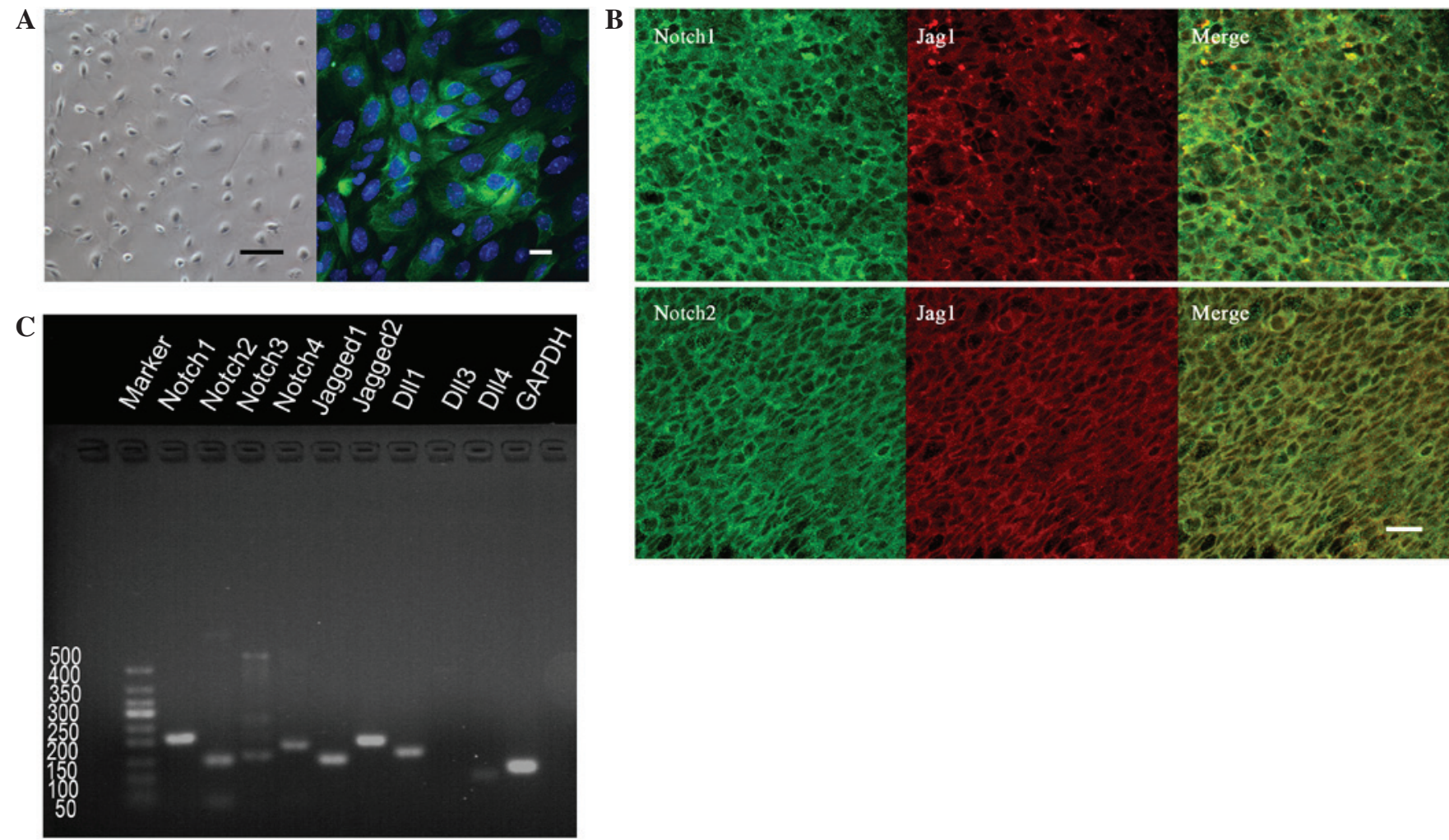

Figure 1. Morphology of cultured normal mouse middle ear epithelium (NMMEE) cells and expression of Notch signaling receptors and ligands. (A) NMMEE cells appeared as a flat monolayer of culture with a polygonal cobblestone-like appearance under a bright-field microscope (left panel; scale bar, $100 \mu \mathrm{m}$ ). Immunocytochemical analysis for pan-cytokeratin (CK) showed that the majority of the cells cultured were pan-CK positive (right panel; scale bar, $20 \mu \mathrm{m}$ ). (B) The majority of cells within the mucosa were Notch1, Notch2 and Jagged1 positive, and were co-localized (scale bar, $20 \mu \mathrm{m}$ ). (C) Reverse transcription polymerase chain reaction indicated that all Notch signaling receptors and ligands were expressed in the NMMEE cells, with the exception of Dll3.

RNA pools were prepared for each DAPT concentration $(2.5$ and $5 \mu \mathrm{M})$ and for the DMSO-treated control cells. Total RNA extraction and cDNA synthesis protocols were performed as described above. PCR primers for Arg2, Hes1, Muc2, Spdef, Spink4 and Tff1 were designed according to the gene sequences obtained from GenBank, as listed in Table II. qPCR was performed using SYBR Green reagent and the Applied Biosystems 7500 Real-time PCR System.

The $2^{-\Delta \Delta C q}$ method was used to quantify the relative differences in mRNA expression levels following DAPT treatment, as previously described (16). For each sample, $\Delta \mathrm{Cq}$ was calculated as the difference in threshold cycle number $(\mathrm{Cq})$ between the target gene and GAPDH. These values were averaged across the samples in a group $(n=3)$. The fold change for each gene following DAPT treatment was determined from $2^{-\Delta \Delta C q}$.

Statistical analysis. All experiments were repeated at least twice, and the results are expressed as the mean \pm standard deviation. The differences between control and experimental groups were evaluated using one-way analysis of variance. $\mathrm{P}<0.05$ was considered to indicate a statistically significant difference.

\section{Results}

Cellular morphology. Primary NMMEE cells demonstrated a flat monolayer of culture with a polygonal cobblestone-like appearance at 5-7 days of culturing, a typical morphology for epithelial cells (Fig. 1A, left panel). The cells exhibited stable growth and maintained the cobblestone-like morphology for up to 10 days. However, the cells showed deterioration of their morphology and significantly swelled and flattened after 10 days.

Cultures of epithelial cells were subjected to immunocytochemistry for pan-CK. The majority of the cells cultured as epithelial cells were pan-CK positive (Fig. 1A, right panel). Thus, it was concluded that epithelial cells were cultured as pure cell populations.

Cellular localization of Notch1, Notch2 and Jagged1 in NMMEE cells. Expression patterns of Notch receptors and their ligands in NMME cells were identified using immunofluorescent staining. As shown in Fig. 1B, marked cell membrane staining of Notch1, Notch2 and Jagged1 was observed, which was co-localized in the majority of cells within the mucosa. These images indicate that Notch receptors and their ligands are expressed in the majority of NMMEE cells.

Expression of Notch receptors and ligands in NMMEE cells. RT-PCR assay was used to detect the expression of Notch receptors and ligands in cultured NMMEE cells. The results indicate that all the receptors (Notch1, Notch2, Notch3 and Notch4) and the majority of the ligands (with the exception of Dl13) were expressed in the cultured NMMEE cells. These results suggest that Notch signaling-associated proteins are present and functional in the mucosa of the mouse middle ear (Fig. 1C). 
A
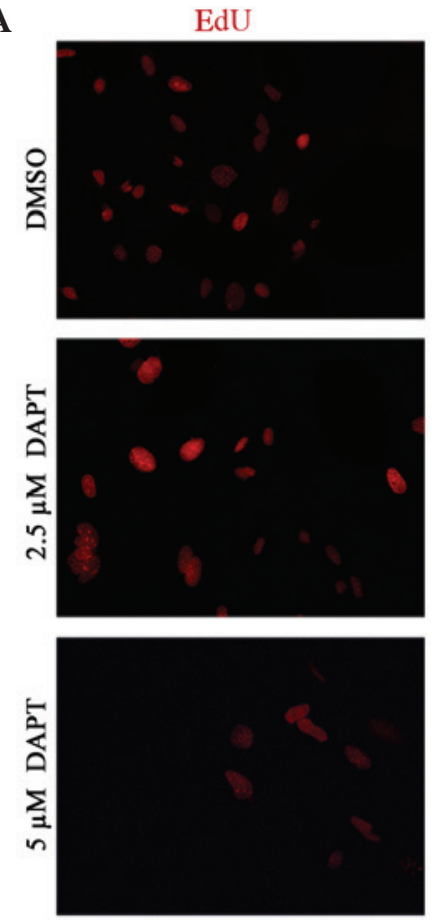

Hoechst
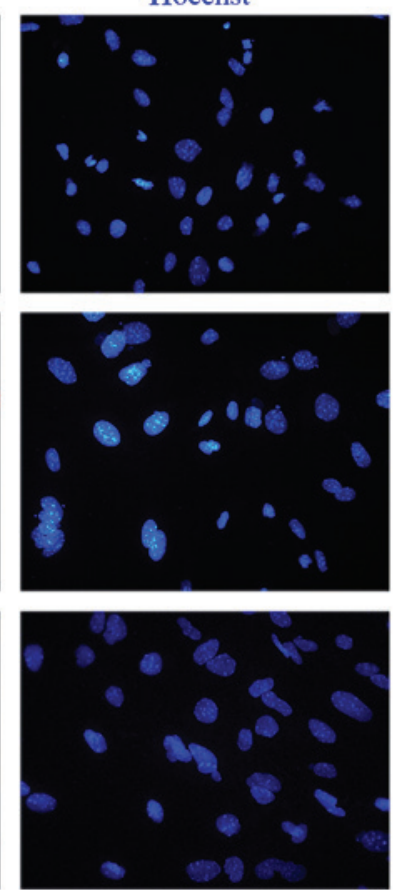

Pan-CK
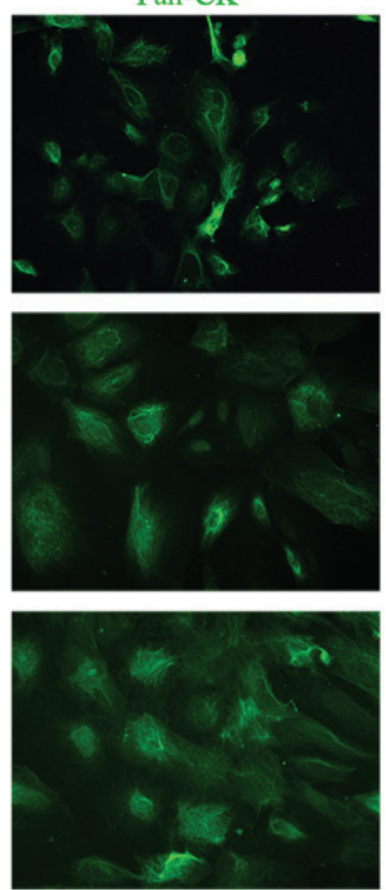
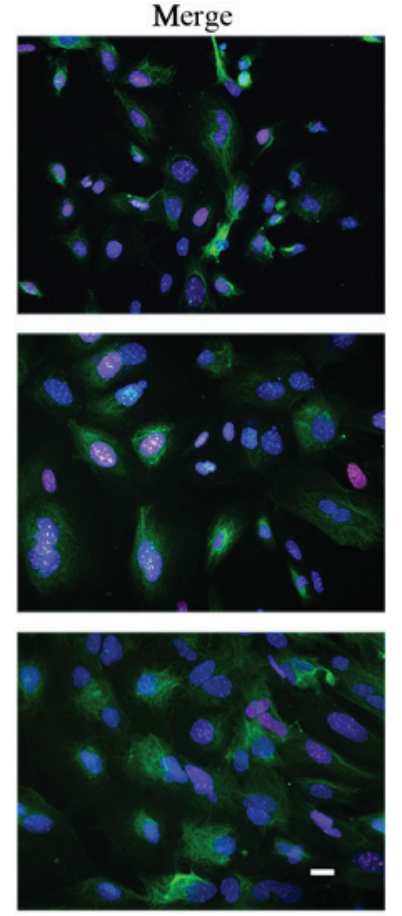

B

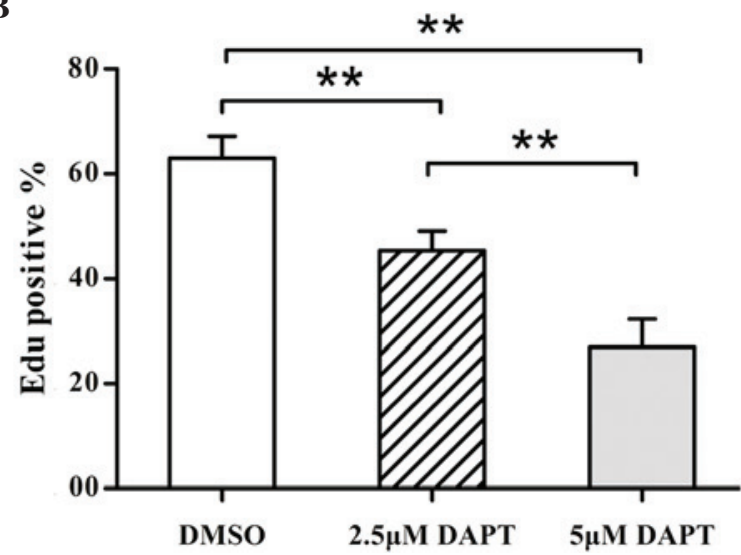

Figure 2. DAPT suppressed cell proliferation in the normal mouse middle ear epithelium (NMMEE) cells by EdU detection. (A) Following treatment with 2.5 or $5 \mu \mathrm{M}$ DAPT, the number of EdU positive NMMEE cells was reduced compared with the control DMSO-treated group. In addition, Hoechst staining was used to identify nuclei and pan-CK staining was used to identify epithelial cell differentiation (scale bar, $20 \mu \mathrm{m}$ ). (B) Quantitative analysis of EdU-positive cells indicated that treatment with 2.5 or $5 \mu \mathrm{M}$ DAPT significantly suppressed the proliferation of NMMEE cells. Data presented as the mean \pm standard deviation ( $\mathrm{n}=6$ per group). ${ }^{* *} \mathrm{P}<0.01$. EdU, 5-ethynyl-2'-deoxyuridine; Pan-CK, pan-cytokeratin; DMSO, dimethyl sulfoxide.

Inhibition of Notch signaling decreases the proliferation of epithelial cells. The enhanced proliferation of middle ear epithelial cells, particularly mucous cells, is a crucial underlying process in the pathogenesis of OM. Therefore, EdU detection was performed in conjunction with immunofluorescence staining for the epithelial cell proliferation marker pan-CK and the apoptosis marker caspase- 3 to evaluate the effect of DAPT on NMMEE cell proliferation and apoptosis in vitro. The results of this experiment were quantitated using image analysis software. Fewer EdU-positive cells were detected among the DAPT-treated NMEE cell samples indicating a reduction in cellular proliferation (Fig. 2A). All of the cultured cells were identified to be pan-CK-positive epithelial cells. The expression of EdU was significantly decreased in DAPT-treated NMMEE cells compared with a DMSO-treated control. Furthermore, the alterations in cellular proliferation appeared to be dose-dependent, since the levels of EdU expression differed significantly between the cells treated with 2.5 DAPT and those treated with $5 \mu \mathrm{M}$ DAPT $(\mathrm{P}<0.01$; Fig. 2A). In addition, the inhibition of $\gamma$-secretase/Notch signaling by DAPT resulted in a dose-dependent reduction in the percentage of cells with positive cell nuclei-associated EdU immunoreactivity (Fig. 2B). The percentages of EdU-positive cells were $63.0 \pm 4.2,45.4 \pm 3.7$ and $27.0 \pm 5.4 \%$ in the cells treated with $0,2.5$ and $5 \mu \mathrm{M}$ DAPT, respectively $(\mathrm{P}<0.01)$. By contrast, the expression of caspase-3 was not found to be increased in the DAPT-treated NMMEE cells compared with the DMSO-treated control cells (data not shown). These data indicate that DAPT inhibited the proliferation of NMMEE cells; however, DAPT is indicated to have no apoptosis-inducing effect on the basis of the immunochemical analysis of caspase-3 expression. 

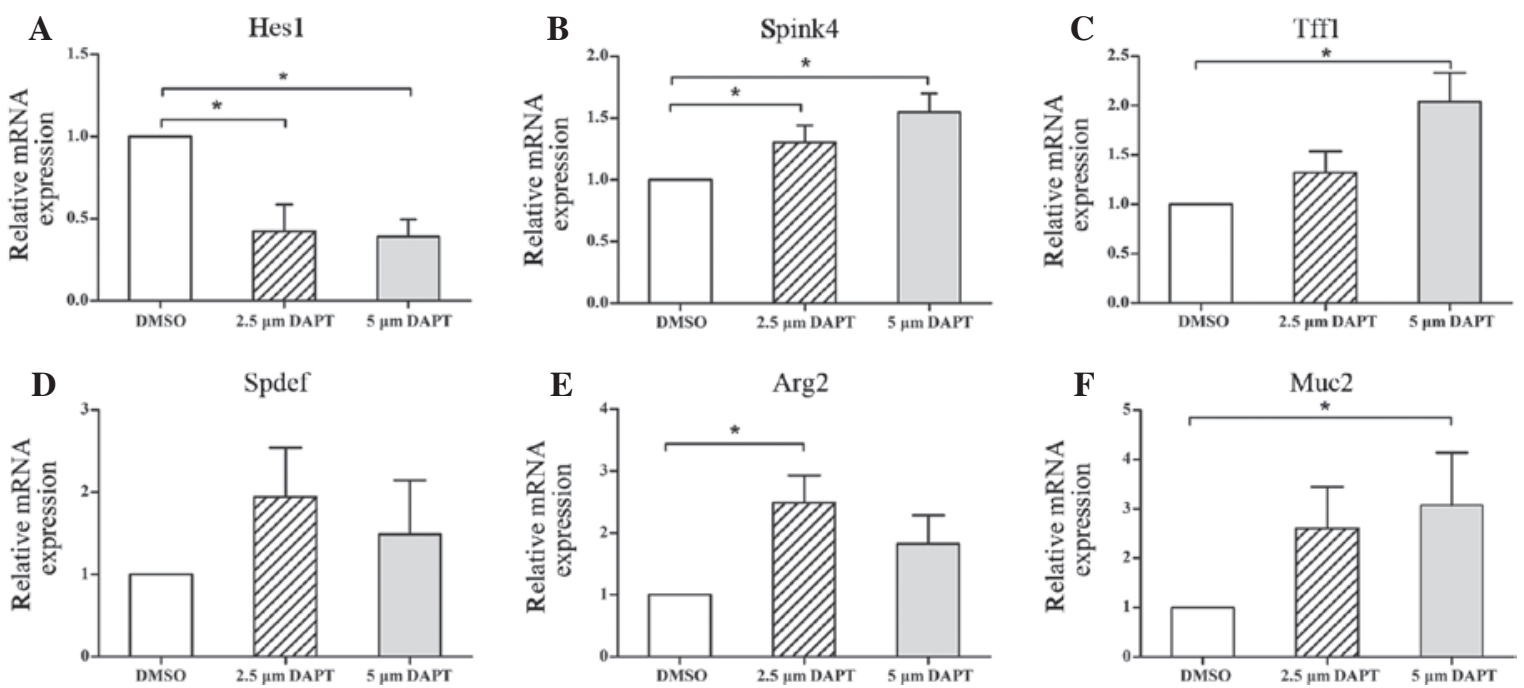

Figure 3. Expression of mucous cell-associated genes in normal mouse middle ear epithelium (NMMEE) cells treated with 2.5 or $5 \mu \mathrm{M}$ DAPT as measured using quantitative polymerase chain reaction. DAPT significantly (A) reduced Hes1 expression and enhanced the mRNA expression levels of (B) Spink4, (C) Tff1, (D) Spdef, (E) Arg2 and (F) Muc2 mRNA. All increases in mRNA expression were statistically significant with the exception of (D) Spdef. Y-axes show relative expression values normalized against glyceraldehyde 3-phosphate dehydrogenase. P-values are for one-way analysis of variance for treatment (DAPT vs. DMSO). "P<0.05. Data presented as the mean \pm standard deviation ( $\mathrm{n}=3$ per group). DMSO, dimethyl sulfoxide.

Collectively, these results indicate that DAPT may exert a therapeutic effect on OM, potentially involving anti-proliferative effects on NMMEE cells.

DAPT activates mucous cell-associated genes in cultured NMMEE cells. Notch signaling triggers mucous cell differentiation in intestinal tissue (9). In colon cancer cells, Notch signal inhibitors are able to activate mucous cell genes (11). In the present study, the effect of the $\gamma$-secretase inhibitor DAPT on NMMEE cell differentiation was investigated. A total of five mucous cell genes (Arg2, Muc2, Spdef, Spink4 and Tff1) were selected due to their effect on intestinal, cancer and airway epithelial cells.

Following subconfluence, NMMEE cells were treated with 2.5 or $5 \mu \mathrm{M}$ DAPT or DMSO (control) for $24 \mathrm{~h}$. To determine the capacity of the treatment to inhibit Notch signaling, Hes1 gene levels were evaluated with and without DAPT treatment. The results indicate that in the 2.5 and $5 \mu \mathrm{M}$ DAPT-treated cells the relative mRNA expression levels of Hes1 were significantly reduced compared with those in the control group $24 \mathrm{~h}$ after treatment with DAPT (Fig. 3A). No statistically significant differences were detected between the two DAPT groups. These observations suggested that 2.5 and $5 \mu \mathrm{M}$ DAPT was able to block Notch signaling within $24 \mathrm{~h}$ of treatment.

To examine the effects that the inhibition of Notch signaling had on the differentiation of NMMEE cells, the expression of Spink4, Tff1, Spdef, Arg2 and Muc2 in NMMEE cells with and without DAPT was investigated. The expression levels of all five mucous cell-associated genes were increased compared with those in the control group at $24 \mathrm{~h}$ (Fig. 3B-F). Among the five mucous cell-associated genes, the expression levels of Muc2 and Arg2 increased the most markedly. The expression levels of all the genes increased in a dose-dependent manner along with the concentration of DAPT, with the exceptions of Spdef and Arg2. The expression levels of Spdef and Arg2 declined in the $5 \mu \mathrm{M}$ DAPT-treated group compared with the $2.5 \mu \mathrm{M}$ DAPT-treated group, although the reduction was not statistically significant. These results suggest that the disruption of Notch signaling leads to a reduction in cell proliferation, with a concomitant increase in mucous cell differentiation.

\section{Discussion}

The mammalian middle ear is an air-filled cavity within the auditory bulla, which has three ossicles suspended within it connecting the eardrum to the inner ear. The cavity is lined by an epithelium, and the auditory tube and eardrum are surrounded with epithelium, which is derived from the first pharyngeal pouch endodermal cells $(15,17,18)$. These cells develop ciliated cells and mucous cells, forming a pseudostratified ciliated columnar epithelium, similar to airway epithelial cells (15). Middle ear epithelial cells are in the frontline against the environment. Mucous cells secrete mucus, and with the cilia constitute a mucociliary system for clearing effusion and debris. The epithelium thickens due to mucous cell metaplasia/hyperplasia in response to disease and inflammation $(4,5)$. In the present study, Notch signaling was inhibited in NMMME cells in order to investigate the mechanism by which middle ear cells proliferate and differentiate, with the aim of elucidating the pathogenesis of mucous cell metaplasia/hyperplasia in OM.

Notch signaling has been well demonstrated in the intestines (9-11). The differentiation of intestinal cells to either an absorptive or secretory cell linage is controlled by the Notch signaling system via lateral inhibition. However, few studies have been published investigating Notch signaling in the middle ear epithelium $(19,20)$. To the best of our knowledge, the present study is the first to reveal the presence of Notch ligands and receptors in NMMEE cells. The mRNA expression of all receptors and ligands investigated was detected in the primary cultured NMMEE cells, with the exception of Dl13, suggesting that Notch signaling may perform certain functions in the epithelium. 
DAPT, a Notch $/ \gamma$-secretase inhibitor, is able to inhibit the NICD cleaved by $\gamma$-secretase, which is subsequently translocated into the nucleus and activates downstream genes $(9,11)$. The results of the present study indicate that the mechanisms underlying the DAPT-mediated suppression of NMMEE cell proliferation involve repressing the activity of the Notch signaling pathway, thereby increasing the expression levels of genes associated with mucous cell differentiation. The expression of the Hes1 gene in cultured NMMEE cells was significantly decreased following treatment with 2.5 or $5 \mu \mathrm{M}$ DAPT for $24 \mathrm{~h}$. The number of EdU positive cells, which represents proliferating cells, decreased as the concentration of DAPT increased. To the best of our knowledge, the present study is the first to demonstrate that Notch signaling is repressed, leading to the suppression of NMMEE cell proliferation by the administration of DAPT. However, no significant differences were detected in the frequency of apoptosis in the DAPT-treated NMMEE cells. These results indicate that DAPT is not able to induce the apoptosis of NMMEE cells in vitro. By contrast, previous studies have clearly demonstrated that $\gamma$-secretase inhibition can enhance the apoptosis of intestinal epithelial cells in vivo $(9,11)$. Therefore, further studies are required to clarify the impact of DAPT within the middle ear epithelium in murine models with OM effusion. In addition to decreased cellular proliferation, DAPT appeared to promote the expression of genes associated with differentiation towards the mucous cell lineage. Further investigation is required to determine by what mechanism these genes influence cell differentiation. On the basis of the present results and the aforementioned previous studies, it is possible that DAPT may exert a therapeutic effect on OM as a result of its suppressive effect on proliferation.

In summary, Notch signaling ligands and receptors are active in the mouse middle ear epithelium. In NMMEE cells primarily cultured in vitro, DAPT was able to inhibit Notch signaling and to thereby suppress the proliferation of the NMMEE cells. Furthermore, DAPT may be able to facilitate the differentiation of epithelial cells into mucous cells. Therefore, the $\gamma$-secretase inhibitor DAPT may provide a specific therapeutic agent for the reversal of multiple pathological processes that are associated with epithelium thickening.

\section{Acknowledgements}

This study was supported by the Major State Basic Research Development Program of China (973 Program; grant no. 2011CB504500 and 2011CB504506), the National Natural Science Foundation of China (grant no. 81420108010, 81000413, 81370022,81570920 and 81200740), the Training Program of the Excellent Young Talents of the Shanghai Municipal Health System (grant no. XYQ2013084) and the Innovation Project of Shanghai Municipal Science and Technology Commission (grant no. 11411952300).

\section{References}

1. Teele DW, Klein JO and Rosner B: Epidemiology of otitis media during the first seven years of life in children in greater Boston: A prospective, cohort study. J Infect Dis 160: 83-94, 1989.

2. Daly KA, Hoffman HJ, Kvaerner KJ, Kvestad E, Casselbrant ML, Homoe P and Rovers MM: Epidemiology, natural history, and risk factors: Panel report from the Ninth International Research Conference on Otitis Media. Int J Pediatr Otorhinolaryngol 74: 231-240, 2010.

3. Ting PJ, Lin CH, Huang FL, Lin MC, Hwang KP, Huang YC, Chiu $\mathrm{CH}$, Lin TY and Chen PY: Epidemiology of acute otitis media among young children: A multiple database study in Taiwan. J Microbiol Immunol Infect 45: 453-458, 2012.

4. Cayé-Thomasen, Hermansson A, Bakaletz L, Hellstrøm S, Kanzaki S, Kerschner J, Lim D, Lin J, Mason K and Spratley J: Panel 3: Recent advances in anatomy, pathology, and cell biology in relation to otitis media pathogenesis. Otolaryngol Head Neck Surg 148 (4 Suppl): E37-E51, 2013.

5. Kerschner JE, Li J, Tsushiya K and Khampang P: Mucin gene expression and mouse middle ear epithelium. Int $\mathbf{J}$ Pediatr Otorhinolaryngol 74: 864-868, 2010.

6. Artavanis-Tsakonas S, Rand MD and Lake RJ: Notch signaling: Cell fate control and signal integration in development. Science 284: 770-776, 1999.

7. Movahedan A, Majdi M, Afsharkhamseh N, Sagha HM, Saadat NS, Shalileh K, Milani BY, Ying H and Djalilian AR: Notch inhibition during corneal epithelial wound healing promotes migration. Invest Ophthalmol Vis Sci 53: 7476-7483, 2012.

8. Ma XB, Jia XS, Liu YL, Wang LL, Sun SL, Song N, Wang EH and Li F: Expression and role of Notch signalling in the regeneration of rat tracheal epithelium. Cell Prolif 42: 15-28, 2009.

9. Kazanjian A, Noah T, Brown D, Burkart J and Shroyer NF: Atonal homolog 1 is required for growth and differentiation effects of notch $/ \gamma$-secretase inhibitors on normal and cancerous intestinal epithelial cells. Gastroenterology 139: 918-928, 2010.

10. Fre S, Huyghe M, Mourikis P, Robine S, Louvard D and Artavanis-Tsakonas S: Notch signals control the fate of immature progenitor cells in the intestine. Nature 435: 964-968, 2005.

11. van Es JH, van Gijn ME, Riccio O, van den Born M, Vooijs M, Begthel H, Cozijnsen M, Robine S, Winton DJ, Radtke F and Clevers H: Notch/gamma-secretase inhibition turns proliferative cells in intestinal crypts and adenomas into goblet cells. Nature 435: 959-963, 2005.

12. Ma A, Boulton M, Zhao B, Connon C, Cai J and Albon J: A role for notch signaling in human corneal epithelial cell differentiation and proliferation. Invest Ophthalmol Vis Sci 48: 3576-3585, 2007.

13. Blanpain C, Lowry WE, Pasolli HA and Fuchs E: Canonical notch signaling functions as a commitment switch in the epidermal lineage. Genes Dev 20: 3022-3035, 2006.

14. Whitsett JA and Kalinichenko VV: Notch and basal cells take center stage during airway epithelial regeneration. Cell Stem Cell 8: 597-598, 2011.

15. Bouras T, Pal B, Vaillant F, Harburg G, Asselin-Labat ML, Oakes SR, Lindeman GJ and Visvader JE: Notch signaling regulates mammary stem cell function and luminal cell-fate commitment. Cell Stem Cell 3: 429-441, 2008.

16. Livak KJ and Schmittgen TD: Analysis of relative gene expression data using real-time quantitative PCR and the 2(-Delta Delta C(T)) method. Methods 25: 402-408, 2001.

17. Thompson $\mathrm{H}$ and Tucker AS: Dual origin of the epithelium of the mammalian middle ear. Science 339: 1453-1456, 2013.

18. Tsuchiya K, Kim Y, Ondrey FG and Lin J: Characterization of a temperature-sensitive mouse middle ear epithelial cell line. Acta Otolaryngol 125: 823-829, 2005.

19. Nakamura Y, Hamajima Y, Komori M, Yokota M, Suzuki M and Lin J: The role of atoh1 in mucous cell metaplasia. Int J Otolaryngol 438609, 2012.

20. Nakamura Y, Komori M, Yamakawa K, Hamajima Y, Suzuki M, Kim Y and Lin J: Math1, retinoic acid, and TNF- $\alpha$ synergistically promote the differentiation of mucous cells in mouse middle ear epithelial cells in vitro. Pediatr Res 74: 259-265, 2013. 